\title{
The Relationship Between Static Standing Posture and Common Pelvic Floor Disorders
}

\author{
P. Zhoolideh'1, F. Ghaderi', Z. Salahzadeh'1, H. Adigozali', M. R. Azghani², \\ M. A. Jafarabadi ${ }^{3,4}$, M. R. Seleme ${ }^{5}$ \\ 1 Department of Physiotherapy, Faculty of Rehabilitation Sciences, Tabriz University of Medical Sciences, Tabriz, Iran \\ 2 Department of Biomechanics, Faculty of Mechanical Engineering, Sahand University of Technology, Tabriz, Iran \\ 3 Road Traffic Injury Research Center, Tabriz University of Medical Sciences, Tabriz, Iran \\ ${ }^{4}$ Department of Statistics and Epidemiology, Faculty of Health, Tabriz University of Medical Sciences, Tabriz, Iran \\ ${ }^{5}$ Department of Pelvic Physiotherapy, Faculty Inspirar, Curitiba, Brazil
}

\section{CORRESPONDING AUTHOR: \\ Fariba Ghaderi \\ Department of Physiotherapy \\ Faculty of Rehabilitation Sciences \\ Tabriz University of Medical Sciences \\ Tabriz, Iran \\ E-mail: ghaderif@tbzmed.ac.ir}

DOI:

10.32098/mltj.01.2021.09

LEVEL OF EVIDENCE: 3B

\begin{abstract}
SUMMARY
Background. Pelvic Floor Disorders (PFDs) include a wide range of diseases. According to biomechanical concepts and previous studies, it can be assumed that there are relations between posture and PFDs but there have been many controversial results. This study aimed to find out if there is any postural change in women with and without common PFDs.

Methods. In this descriptive cross-sectional study, a total of 47 women with PFDs and 47 matched controls (aged 25-45), participated. Static standing posture was measured by photogrammetry and flexible ruler for spinal curvature.

Results. In the case group, the lumbar lordosis $(\mathrm{p}=0.002)$ and $\mathrm{Q}$ angle $(\mathrm{p}<0.001)$ significantly decreased while the thoracic kyphosis $(\mathrm{p}=0.002)$, foot pronation angle $(\mathrm{p}<0.001)$ and sagittal shoulder angle $(\mathrm{p}=0.002)$ increased significantly in comparison with the control.

Conclusions. This study showed that some of the postural changes in the spine, shoulder, pelvis, and lower limbs were prone to be associated with common PFDs (urinary incontinence, fecal incontinence, pelvic organ prolapses, and chronic pelvic pain syndrome).

\section{KEY WORDS}

Pelvic Floor Disorders; Urinary Incontinence (UI); Fecal Incontinence (FI); Pelvic Organ Prolapses (POP); Chronic Pelvic Pain Syndrome (CPP); posture; photogrammetry; flexible ruler.
\end{abstract}

\section{INTRODUCTION}

Pelvic Floor Dysfunctions (PFDs) result in a wide range of problems such as urinary incontinence (UI), fecal incontinence $(\mathrm{FI})$, pelvic organ prolapses (POP), chronic pelvic pain syndrome (CPP), and dyspareunia (1). These dysfunctions affect the quality of life and social relations of patients and are prevalent in different societies being higher in women than men (2). In a study conducted in Iran (2012), $42 \%$ of women showed at least one kind of PFDs (3) GC. A probable association between bony and visceral structures of the pelvic floor has been mentioned before (4). Therefore, in hypothesis, any postural abnormality may alter the performance of pelvic muscles and can lead to PFDs.

Some of the previous studies investigated the association of posture and PFDs (4-8). Life style and the environment can alter the length-tension of muscles in the pelvic, low back and hip area so it can change the posture and affect the visceral and muscular structures of the pelvic floor (9).

With this hypothesis, it can be assumed that posture may influence the activation of Pelvic Floor Muscles (PFM) and their coordination with other trunk muscles. There are some investigations which studied the relations between different temporary postural changes and PFM activation in healthy subjects and in subjects with PFDs (10-13).

Other studies have been conducted with this assumption that there is such a relationship, and they have examined the effects of postural global exercises on the treatment of urinary incontinence, and concluded that these exercises can be effective in treating the symptoms of these patients $(14,15)$. The relationship between postural changes and 
PFDs has been shown in some studies. Mattox et al. (2000), Nguyen et al. (2000), and Sayah melli et al. (2007) showed a decrease in lumbar lordosis and an increase in thoracic kyphosis using different measurement methods in PFDs $(4,5,7)$. Nygaard et al. (1996), also found that flat foot was associated with a risk of urinary incontinence (7). Also, protracted shoulder and forward head posture in patients with chronic pelvic pain have also been reported $(14,16)$. However, there are controversial studies that did not find any significant correlation between the postural variables and PFDs (17-19).

Investigation of the relationship between postural changes and PFDs can improve the assessment and treatment protocols in patients with PFDs. Although these changes may not be the primary cause of PFDs, they can lead to increasing pain and symptoms in these patients (9). Besides, therapists can use these findings for early preventions and multi-disciplinary treatment of such a multi-factorial disorder.

Therefore, based on the available and controversial evidence, this analytical descriptive cross-sectional study was conducted to evaluate the various aspects of static standing posture in women with and without common PFDs using photogrammetry to find if there is any probable change in bony structure and spinal curvatures.

\section{MATERIALS AND METHODS}

\section{Participants}

In this descriptive cross-sectional study, 47 women with common PFDs and 47 matched women without any PFDs (aged 25-45) participated. Available patients with UI, $\mathrm{POP}$ or CPP who were diagnosed by a gynecologist and referred to the Physiotherapy Clinic were included in the case group. The control group was matched in terms of age, number of births and Body Mass Index (BMI) with the case group.

Subjects with menopause, pregnancy, history of spinal, hip, knee or foot surgery, pelvic and abdominal surgery (including cesarean section), smoking, chronic cough, history of respiratory diseases, neurological and connective tissue diseases, severe depression (Beck depression score $>40$ ), osteoporosis (self-reported) and individuals with BMI $>30$ were excluded from the study.

\section{Ethical approval}

This study was approved by the Regional Committee for Medical Ethics of Tabriz University of Medical Sciences (approval number: IR. TBZMED.1395.11). An informed consent was approved also by this committee. All the proce- dure was explained to all of the participants and if they agreed with the whole procedure, the written informed consent was taken from all of them. This study meets the ethical standards of the journal (20).

\section{Outcomes}

\section{Depression score}

All the participants completed a demographic questionnaire and a Beck depression questionnaire. A valid and reliable Persian version of the Beck depression questionnaire was used (21) to determine the depression score of the participants.

\section{Postural paremeters}

Different postural parameters including:

1. lumbar lordosis and thoracic kyphosis;

2. A) head sagittal tilt angle (the angle between lines cross canthus, tragus and horizontal), B) craniovertebral angle (the angle between lines cross tragus, spinal process of C7 and horizontal), C) rounded shoulder angle (the angle between lines cross spinal process of $\mathrm{C} 7$, lateral of shoulder and horizontal), D) Lumbar curve angle (the angle between lines cross spinal process of T12, L1 and anterior superior iliac spine (ASIS), posterior superior iliac spine (PSIS)), E) pelvic inclination to femur angle (the angle between lines cross ASIS, PSIS and greater trochanter), F) sagittal pelvic tilt (the angle between lines cross ASIS, PSIS and horizontal), G) knee alignment (the angle between lines cross greater trochanter, lateral point of knee and lateral malleolus), H) lateral ankle alignment (the angle between lines cross lateral malleolus, head of fibula and horizontal) in lateral view (figure 1);

3. A) head coronal tilt (the angle between line cross lobule of both ears and horizontal), B) coronal shoulder angle (the angle between left and right corocoid process), C) coronal pelvic tilt (the angle between lines cross both ASIS and horizontal), D) Quadriceps angle (the angle between lines cross ASIS, medial point of patella and tibial tuberosity) in anterior view (figure 2);

4. A) scapular alignment (the angle between lines cross inferior angle of both scapula and horizontal), B) coronal pelvic tilt (the angle between lines cross both PSIS and horizontal), C) pronation/supination of foot (the angle between lines cross medial and lateral malleolus and horizontal) in posterior view (figure 3 ).

\section{Procedure}

In this descriptive cross-sectional study, all evaluations carried out by same trained physiotherapist verified by the group leader (F. G.). It should be noted that all assessments were performed between $11 \mathrm{am}$ to $4 \mathrm{pm}$. All stages of the 


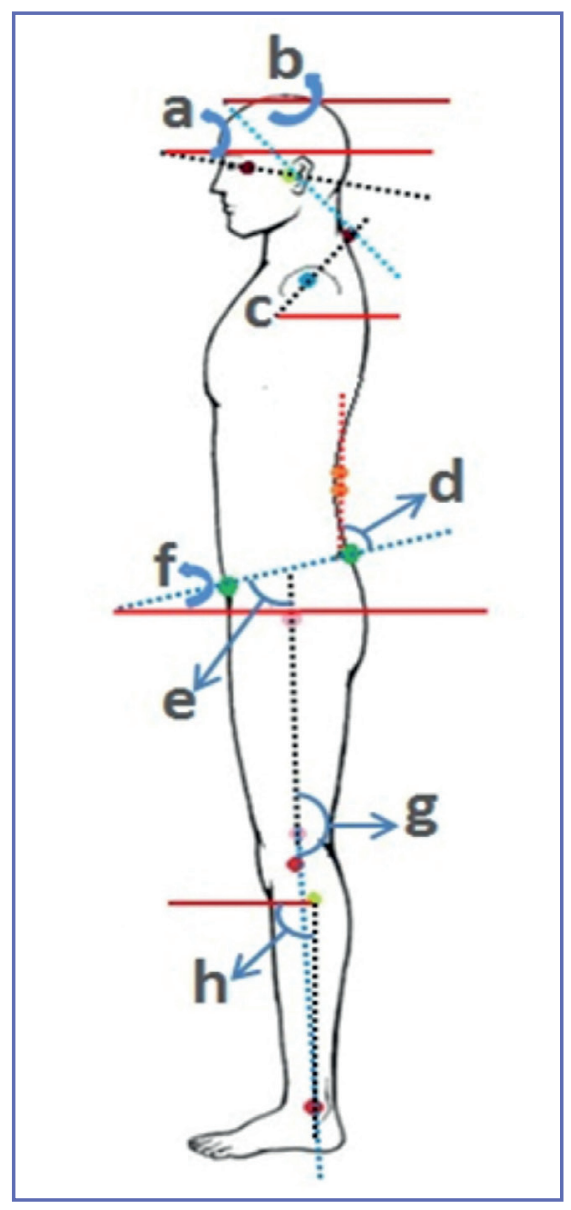

Figure 1. Landmarks and angles using photogrammetry method (lateral view): A) head sagittal tilt angle, B) craniovertebral angle (angle between lines cross tragus, spinal process of C7 and horizontal), C) rounded shoulder angle, D) lumbar curve angle, E) pelvic inclination to femur angle, F) sagittal pelvic, G) lateral knee, H) lateral ankle alignment.

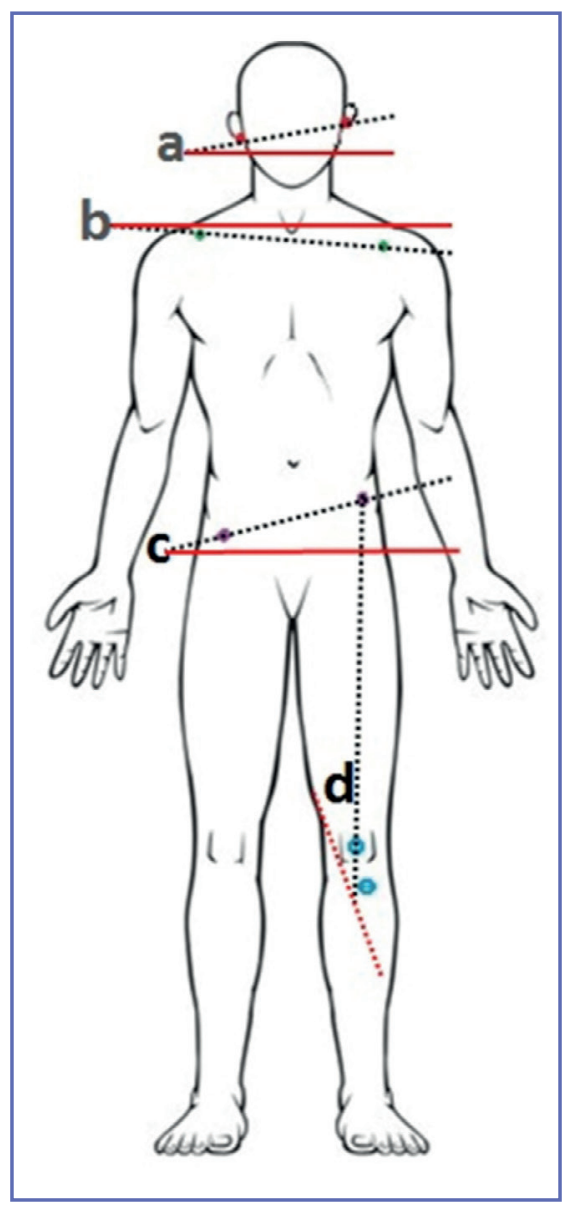

Figure 2. Landmarks and angles using photogrammetry method (anterior view): A) head coronal angle, B) coronal shoulder angle, C) coronal pelvic tilt, D) quadriceps angle.

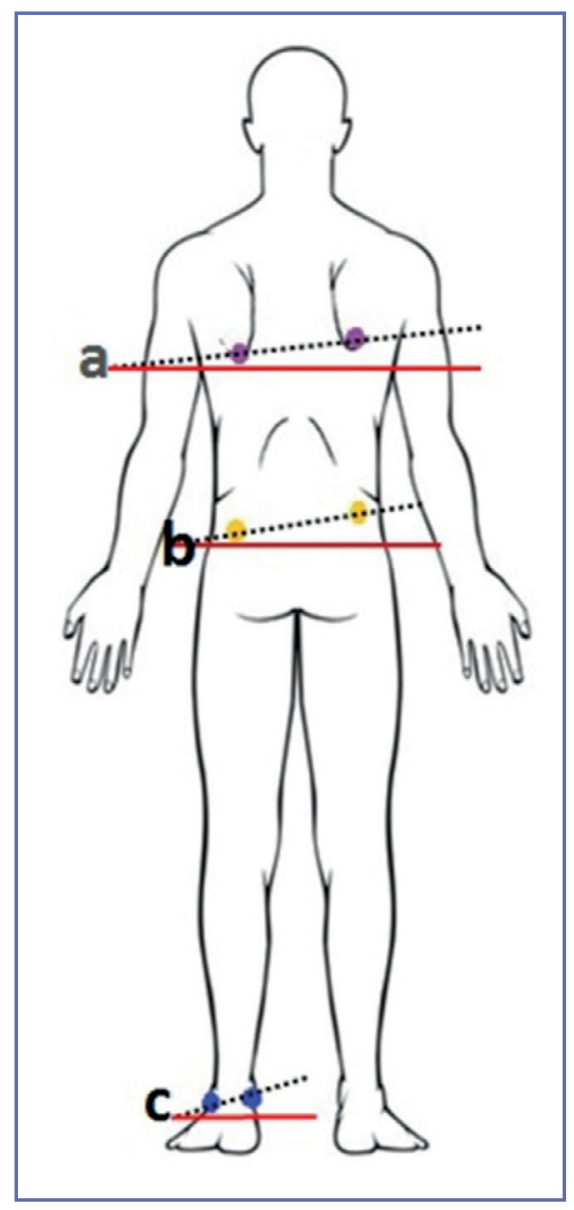

Figure 3. Landmarks and angles using photogrammetry method (posterior view): A) scapula alignment, B) coronal pelvic tilt, C) pronation/supination of foot. study were performed under the supervision of an experienced gynecologist.

The curvature of the thoracic (from T2 to T12) and lumbar spines (from L1 to $\mathrm{S} 1$ ) were evaluated using a flexible ruler separately (Forsun 24 Inch $(60 \mathrm{~cm})$ Flexible Curve Ruler Flex Design Rule). The participants were asked to attain their habitual posture by using a self-balanced position (22). Then the flexible ruler was placed on the landmarks marked on the thoracic and lumbar spine separately. Finally, curved ruler transferred on paper and thoracic and lumbar spines were evaluated (22). Photogrammetry was done using a digital camera (Canon power shot sx $30 \mathrm{hs}$ ) with a height of $90 \mathrm{~cm}$ and a distance of $3 \mathrm{~m}$ from the subject. Reflex markers were located in anatomical landmarks and photogrammetric photos were taken from the anterior, posterior and the lateral left views. The anatomical landmarks included: the lobule of ears, both anterior superior iliac spine (ASIS), left and right coracoid process tibial tuberosity, the medial point of patella in the anterior view; and the inferior angle of scapula, left and right Posterior superior iliac spine (PSIS), medial and lateral malleolus of the left foot in posterior view; and tragus, canthus, lateral point of shoulder, spinal process of C7, L1 and T12, left ASIS and PSIS, medial point of knee joint, lateral malleolus, lateral epicondyle of femur, head of fibu- 
la, greater trochanter of femur. All postural angles were measured using AutoCAD 2010. The participants were asked to attain their habitual posture by using a self-balanced position before taking the photos (22).

The inter and intra tester reliability was checked by examining 10 healthy controls and 10 patients in two days by two different examiners for both photogrammetry and flexible ruler method. Both examiners were experienced physiotherapists who performed the assessments under the supervision of the group leader.

\section{Statistical analysis}

The SPSS statistics software 21.0 was used to analyze the results. The skewness and kurtosis indexes were checked for normality. Kolmogrov-Smirnov test was also checked for normality. Independent T-test for variables with normal distribution to compare the results between the two groups. Intercorrelation coefficient (ICC) was used to determine inter and intra tester reliability of the photogrammetry.

\section{RESULTS}

The inter and intra tester reliability were checked between the two different testers in two different days. Inter tester $(\mathrm{ICC}=0.88 \pm 0.09)$ and intra tester $(\mathrm{ICC}=0.95 \pm 0.02)$ reliability were acceptable.
There was no significant difference between the two groups in terms of age and BMI ( $p>0.05)$ (table I). Frequency distribution of participating women showed 30.9 percent UI, 9.9 percent POP, 7.4 percent CPP and 2.1 percent more than one disorders. The case group showed higher scores in the Beck depression test $(10.7 \pm 5.08)$ in comparison with the controls $(6.72 \pm 2.7)(\mathrm{p}=0.001)$.

Kolmogrov-Smirnov test showed normal distribution in all variables. Variables with a significant difference between the two groups were: lumbar lordosis was significantly decreased $(\mathrm{p}=0.002)$ in the case group $(31.29 \pm 8.9$ degrees $)$ compared to the control group $(37.06 \pm 9.03$ degrees $)$ and thoracic kyphosis was significantly increased in case group (34.8 \pm 4.8) compared to control group (31.9 \pm 3.7$)$ (table II).

The angle of the quadriceps was significantly decreased in case group (15.7 \pm 6.4 degrees) compared to control group $(22.4 \pm 5.4$ degrees $)(\mathrm{p}<0.001)$. Foot pronation angle in

Table I. Participant's demographic information.

\begin{tabular}{lccc}
\hline \multirow{2}{*}{ Variable } & $\begin{array}{c}\text { Case } \\
\text { group }(\mathrm{N}: 47)\end{array}$ & $\begin{array}{c}\text { Control } \\
\text { group (N:47) }\end{array}$ & \multirow{2}{*}{ P-value } \\
\cline { 2 - 3 } & Mean \pm SD & Mean \pm SD & \\
\hline Age & $37.74 \pm 6.29$ & $37.43 \pm 6.17$ & 0.805 \\
\hline BMI & $25.83 \pm 2.60$ & $25.87 \pm 2.30$ & 0.901 \\
\hline
\end{tabular}

Table II. Results of independent T-test analysis in case and control groups.

\begin{tabular}{|c|c|c|c|}
\hline \multirow{2}{*}{ Variable } & Case group (N: 47) & Control group (N: 47) & \multirow{2}{*}{ P-value } \\
\hline & Mean \pm SD & Mean \pm SD & \\
\hline Beck depression score & $10.70 \pm 5.08$ & $6.02 \pm 2.70$ & $<0.001 *$ \\
\hline Quadriceps angle (degree) & $15.70 \pm 6.40$ & $22.40 \pm 5.40$ & $<0.001 *$ \\
\hline Pronation/Supination of foot (degree) & $6.90 \pm 7.10$ & $12.20 \pm 4.30$ & $<0.001^{*}$ \\
\hline Rounded shoulder angle (degree) & $-0.40 \pm 9.30$ & $-10.40 \pm 9.70$ & $0.002^{*}$ \\
\hline Lumbar Lordosis (degree) & $31.29 \pm 8.90$ & $37.06 \pm 9.03$ & $0.002 *$ \\
\hline Thoracic kyphosis (degree) & $34.80 \pm 4.80$ & $31.9 \pm 3.70$ & $0.002 *$ \\
\hline Head sagittal tilt (degree) & $15.90 \pm 8.10$ & $18.4 \pm 6.60$ & 0.107 \\
\hline Craniovertebral Angle (degree) & $46.50 \pm 6.70$ & $47.60 \pm 5.50$ & 0.362 \\
\hline Pelvic inclination to femur angle & $-18.09 \pm 5.40$ & $-17.06 \pm 5.60$ & 0.374 \\
\hline Pelvic sagittal tilt (degree) & $-18.09 \pm 5.40$ & $-17.06 \pm 5.60$ & 0.374 \\
\hline Knee alignment (extension/flexion) (degree) & $184.80 \pm 3.80$ & $184.40 \pm 2.80$ & 0.563 \\
\hline Lateral ankle alignment (degree) & $97.60 \pm 3.90$ & $97.50 \pm 2.70$ & 0.880 \\
\hline Head coronal tilt (degree) & $-0.80 \pm 2.60$ & $-0.60 \pm 1.90$ & 0.655 \\
\hline Pelvic Coronal tilt ant view (degree) & $-0.60 \pm 3.30$ & $-0.30 \pm 2.20$ & 0.774 \\
\hline Pelvic Coronal tilt posterior view (degree) & $-0.10 \pm 3.00$ & $0.40 \pm 1.09$ & 0.313 \\
\hline Scapular alignment (degree) & $-0.70 \pm 2.40$ & $-0.40 \pm 1.90$ & 0.457 \\
\hline
\end{tabular}

The negative amount indicates the left side of the body is higher. *Indicates significant difference between both groups. 
case group $(6.9 \pm 7.1$ degrees $)$ was significantly lower in comparison with control group $(12.2 \pm 4.3$ degrees $)$ ( $\mathrm{p}<$ $0.001)$. The sagittal shoulder angle in the case group $(-0.4 \pm$ 9.3 degrees) was significantly lower compared to the control group $(10.4 \pm 9.7$ degrees $)(\mathrm{p}=0.002)$.

Other postural variables (head sagittal tilt angle, craniovertebral angle, pelvic inclination to femur angle, sagittal pelvic tilt, knee alignment, lateral ankle alignment, head coronal tilt, coronal pelvic tilt, scapular alignment) that we evaluated did not show any significant difference between the case and control groups.

\section{DISCUSSION}

This study demonstrated that the women with PFDs had higher depression score, rounded shoulder, thoracic hyper kyphosis, lumbar hypo lordosis, knee Varus and foot pronation in comparison to the control group. It would be recommended that physiotherapists evaluate the patient's posture and faulty postures as well as PFDs.

Higher depression score in patients with PFD was shown as PFD are associated with decrease in the quality of life and also have high impact on social relationships and self-image $(23,24)$. The increase in thoracic kyphosis and decrease in lumbar lordosis in patients with PFDs are one of the findings of this study which are in line with most of the previous studies (4-6). The decrease in lumbar lordosis is one of the factors that increased intra-abdominal pressure, led to compressed pelvic floor structures which could lead to some changes in soft tissues around the pelvic and results in pelvic mal-alignment $(5,25)$. In fact, if these forward and backward curvatures maintain their normal structure, they are among the important supportive levels in absorbing intra-abdominal pressure before they reach the pelvic structures. There are limited studies about postural abnormalities in patients with PFDs.

Capson et al. (2011) studied the role of lumbopelvic posture in PFM activation in healthy subjects and showed higher resting PFM activity in hypo lordotic posture (10). Ptaszkowski et al. (2017) showed the increase of PFM synergistic muscles activation in posterior pelvic tilt in incontinent patients (11). These two studies investigated the effects of a temporary posture on PFM activation, but habitual posture is different because compensations during time can change the activation pattern of muscles. However, increasing PFM activation during a day in a habitual posture may result in fatigue and weakness of the muscles and ultimately PFDs.
There was one controversial study (2016) which reported no significant thoracic and lumbar curvature changes in patients with PFDs using spinal $x$-rays. But there was no time limitation and a specific position for taking $\mathrm{X}$-rays so it could be the point of these controversial results (17).

On the other hand, studies have shown that an increased rate of depression can be another reason for the kyphotic posture and rounded shoulder in PFD patients (26).

By changing the spinal curvatures and according to the closed kinematic chain rule, posterior pelvic tilt in relation to hypo lordosis and hyper kyphosis may lead to a pronated foot (27) as was seen in the present study. The ankle pronation may cause internal rotation of the tibia and lateral rotation of the femur this can reduce the angle of the quadriceps and knee Varus in PFD patients (28). The results of Montenegro et al. (2009), Miranda et al. (2009) and Ansarian et al. (2014) about the knee and ankle alignment of women with PFDs are inconsistent with this study. It may be because of the way of measuring postural changes; previous studies used observational methods for evaluating posture $(16,18,29)$.

As with any other studies, the current study might have some limitations, including the small sample size and no golden standards for the evaluation of posture like x-rays. Furthermore, based on the findings of this study, more variables might differ in patients with PFDs, although due to our limitations in measurement instruments such as: using only one camera to capture, probable human errors in evaluations, we were not able to identify other probable changes. It would have been appropriate to correlate these data with electromyography or other muscle activation tests and findings of Magnetic resonance imaging (MRI) as these methods were not used in our study.

\section{CONCLUSIONS}

This study showed that some of the postural changes in the spine, shoulder, pelvis, and lower limbs are associated with PFDs, so therapists should take this aspect of PFDs into account for the prevention and treatment of the patients.

\section{ACKNOWLEDGEMENTS}

We gratefully acknowledge the support and generosity of all patients who helped us with our study.

\section{CONFLICT OF INTERESTS}

The authors declare that they have no conflict of interests. 


\section{REFERENCES}

5. Weber A, Abrams P, Brubaker L, Cundiff G, Davis G, Dmochowski R. The standardization of terminology of female pelvic organ prolapse and pelvic floor dysfunction. Int Urogynecol J Pelvic Floor Dysfunct 2001;12:178-86.

6. Bo K, Frawley HC, Haylen BT, Morin M, Shelly E. Response to comment on the IUGA/ICS joint report on the terminology for the conservative and nonpharmacological management of female pelvic floor dysfunction. Int Urogynecol J 2017;28(8):1269-70.

7. Eftekhar T, Ghanbari Z, Kalantari F, Shariat M, Haghollahi F. The frequency of pelvic floor dysfunctions and their risk factors in women aged 40-55. J Family Reprod Health 2012;6(2):59-64.

8. Melli MS, Alizadeh M. Abnormal spinal curvature as a risk factor for pelvic organ prolapse. PJBS 2007;10(23):4218-23.

9. Mattox TF, Lucente V, McIntyre P, Miklos JR, Tomezsko J. Abnormal spinal curvature and its relationship to pelvic organ prolapse. Am J Obstet Gynecol 2000;183(6):1381-4.

10. Nguyen JK, Lind LR, Choe JY, McKindsey F, Sinow R, Bhatia NN. Lumbosacral spine and pelvic inlet changes associated with pelvic organ prolapse. Obstet Gynecol 2000;95(3):332-6.

11. Nygaard IE, Glowacki C, Saltzman CL. Relationship between foot flexibility and urinary incontinence in nulliparous varsity athletes. Obstet Gynecol 1996;87(6):1049-51.

12. Zhoolideh P, Ghaderi F, Salahzadeh Z. Are There any Relations Between Posture and Pelvic Floor Disorders? A Literature Review. Crescent J Med Biol Sci 2017;4:153-9.

13. King P, Myers C, Ling F, Rosenthal R. Musculoskeletal factors in chronic pelvic pain. J Psychosom Obstet Gynaecol 1991;12(sup1):87-98.

14. Capson AC, Nashed J, Mclean L. The role of lumbopelvic posture in pelvic floor muscle activation in continent women. J Electromyogr Kinesiol 2011;21(1):166-77.

15. Ptaszkowski K, Zdrojowy R, Slupska L, et al. Assessment of bioelectrical activity of pelvic floor muscles depending on the orientation of the pelvis in menopausal women with symptoms of stress urinary incontinence: continued observational study. Eur J Phys Rehabil Med 2017;53(4):564-74.

16. Chen H-L, Lin Y-C, Chien W-J, Huang W-C, Lin H-Y, Chen P-L. The effect of ankle position on pelvic floor muscle contraction activity in women. J Urol 2009;181(3):1217-23.

17. Kannan P, Winser S, Goonetilleke R, Cheing G. Ankle positions potentially facilitating greater maximal contraction of pelvic floor muscles: A systematic review and meta-analysis. Disabil Rehabil 2019;41(21):2483-91.

18. Fozzatti MCM, Palma P, Herrmann V, Dambros M. Impacto da reeducação postural global no tratamento da incontinência urinária de esforço feminina. Rev Assoc Med Bras 2008;54(1):17-22.

19. Fozzatti C, Herrmann V, Palma T, Riccetto CL, Palma PC. Global Postural Re-education: an alternative approach for stress urinary incontinence? Eur J Obstet Gynecol Reprod Biol 2010;152(2):218-24.

20. Montenegro ML, Mateus-Vasconcelos EC, e Silva JCR, dos Reis FJC, Nogueira AA, Poli-Neto OB. Postural changes in women with chronic pelvic pain: a case control study. BMC musculoskeletal disorders 2009;10(1):82.

21. Meyer I, McArthur TA, Tang Y, McKinney JL, Morgan SL, Richter HE. Pelvic floor symptoms and spinal curvature in women. Female Pelvic Med Reconstr Surg 2016;22(4):219.

22. Ansarian M, Gharamaleki ME, Ghaderi F, Ghafari ME, Asaadi N. The relationship between stress urinary incontinence in women and flat foot by using two methods. Adv Environ Biol 2014:377-86.

23. 19. Stein TA, Kaur G, Summers A, Larson KA, DeLancey JO. Comparison of bony dimensions at the level of the pelvic floor in women with and without pelvic organ prolapse. Am J Obstet Gynecol 2009;200(3):241. e1-. e5.

24. Padulo J, Oliva F, Frizziero A, Maffulli N. Basic principles and recommendations in clinical and field science research: 2018 update. MLTJ 2018;8(3):305-7.

25. Ghassemzadeh H, Mojtabai R, Karamghadiri N, Ebrahimkhani N. Psychometric properties of a Persian-language version of the Beck Depression Inventory-Second edition: BDI-II-PERSIAN. Depress Anxiety 2005;21(4):185-92.

26. Greenfield B, Catlin P, Coats P. Posture in patients with shoulder overuse injuries and healthy individuals. Orthop Sports Phys Ner 1995;21:287-95.

27. Vrijens D, Berghmans B, Nieman F, van Os J, van Koeveringe G, Leue C. Prevalence of anxiety and depressive symptoms and their association with pelvic floor dysfunctions-A cross sectional cohort study at a Pelvic Care Centre. Neurourol Urodynam 2017;36(7):1816-23.

28. Pizarro-Berdichevsky J, Hitschfeld MJ, Pattillo A, et al. Association between pelvic floor disorder symptoms and QoL scores with depressive symptoms among pelvic organ prolapse patients. ANZJOG 2016;56(4):391-7.

29. Bisciotti GN, Auci A, Cena E, et al. Potential MRI findings associated with inguinal hernia and inguinal canal posterior wall weakness in athletes. MLTJ 2018;8(2):290-304.

30. Canales JZ, Cordás TA, Fiquer JT, Cavalcante AF, Moreno RA. Posture and body image in individuals with major depressive disorder: a controlled study. Braz J Psychiatr 2010;32(4):375-80.

31. Handa VL, Lockhart ME, Kenton KS, et al. Magnetic resonance assessment of pelvic anatomy and pelvic floor disorders after childbirth. Int Urogynecol J 2009;20(2):133-9.

32. Levine D, Whittle MW. The effects of pelvic movement on lumbar lordosis in the standing position. J Orthop Sports Phys Ther 1996;24(3):130-5.

33. Miranda R, Schor E, Girão MJBC. Postural evaluation in women with chronic pelvic pain. Rev Bras de Ginecol e Obstet 2009;31(7):353-60. 